\title{
HYERS-ULAM-RASSIAS STABILITY OF GENERALIZED DERIVATIONS
}

\author{
MOHAMMAD SAL MOSLEHIAN
}

Received 10 September 2005; Revised 21 March 2006; Accepted 4 April 2006

The generalized Hyers-Ulam-Rassias stability of generalized derivations on unital Banach algebras into Banach bimodules is established.

Copyright (c) 2006 Hindawi Publishing Corporation. All rights reserved.

\section{Introduction}

One of the interesting questions in the theory of functional equations concerning the problem of the stability of functional equations is as follows: when is it true that a mapping satisfying a functional equation approximately must be close to an exact solution of the given functional equation?

The first stability problem was raised by Ulam during his talk at the University of Wisconsin in 1940 [18].

Given a group $G_{1}$, a metric group $\left(G_{2}, d\right)$, and a positive number $\varepsilon$, does there exist a $\delta>0$ such that if a mapping $f: G_{1} \rightarrow G_{2}$ satisfies the inequality $d(f(x y), f(x) f(y))<\delta$ for all $x, y \in G_{1}$, then there exists a homomorphism $T: G_{1} \rightarrow G_{2}$ such that $d(f(x), T(x))<$ $\varepsilon$ for all $x \in G_{1}$ ?

Ulam's problem was partially solved by Hyers in 1941 in the context of Banach spaces with $\delta=\varepsilon$ as shown below [7].

Suppose that $E_{1}, E_{2}$ are Banach spaces and $f: E_{1} \rightarrow E_{2}$ is a mapping for which there exists $\varepsilon>0$ such that $\|f(x+y)-f(x)-f(y)\|<\varepsilon$ for all $x, y \in E_{1}$. Then there is a unique additive mapping $T: E_{1} \rightarrow E_{2}$ defined by $T x=\lim _{n \rightarrow \infty}\left(f\left(2^{n} x\right) / 2^{n}\right)$ such that $\| f(x)-$ $T(x) \|<\varepsilon$ for all $x \in E_{1}$.

Now assume that $E_{1}$ and $E_{2}$ are real normed spaces with $E_{2}$ complete, $f: E_{1} \rightarrow E_{2}$ is a mapping such that for each fixed $x \in E_{1}$ the mapping $t \mapsto f(t x)$ is continuous on $\mathbb{R}$, and that there exist $\varepsilon \geq 0$ and $p \neq 1$ such that

$$
\|f(x+y)-f(x)-f(y)\| \leq \varepsilon\left(\|x\|^{p}+\|y\|^{p}\right)
$$

for all $x, y \in E_{1}$. 
It was shown by Rassias [15] for $p \in[0,1)$ (and indeed $p<1$ ) and Gajda [4] following the same approach as in [15] for $p>1$ that there exists a unique linear map $T: E_{1} \rightarrow E_{2}$ such that

$$
\|f(x)-T(x)\| \leq \frac{2 \varepsilon}{\left|2^{p}-2\right|}\|x\|^{p}
$$

for all $x \in E_{1}$. This phenomenon is called Hyers-Ulam-Rassias stability. It is shown that there is no analogue of Rassias result for $p=1$ (see $[4,17]$ ). [5].

In 1994, a generalization of the Rassias theorem was obtained by Găvruţa as follows

Suppose $(G,+)$ is an abelian group, $E$ is a Banach space, and that the so-called admissible control function $\varphi: G \times G \rightarrow[0, \infty)$ satisfies

$$
\tilde{\varphi}(x, y):=\frac{1}{2} \sum_{n=0}^{\infty} 2^{-n} \varphi\left(2^{n} x, 2^{n} y\right)<\infty
$$

for all $x, y \in G$. If $f: G \rightarrow E$ is a mapping with

$$
\|f(x+y)-f(x)-f(y)\| \leq \varphi(x, y)
$$

for all $x, y \in G$, then there exists a unique mapping $T: G \rightarrow E$ such that $T(x+y)=T(x)+$ $T(y)$ and $\|f(x)-T(x)\| \leq \tilde{\varphi}(x, x)$ for all $x, y \in G$.

Since then several stability problems of various functional equations have been investigated by many mathematicians. The reader is referred to $[3,16]$ for a comprehensive account of the subject.

Generalized derivations first appeared in the context of operator algebras [8]. Later, these were introduced in the framework of pure algebra [6]. There is also another generalization of the notion of derivation which is called $(\sigma, \tau)$-derivation (cf. [9]).

Let $\mathscr{A}$ be an algebra and let $\mathscr{X}$ be an $\mathscr{A}$-bimodule. A linear mapping $\mu: \mathscr{A} \rightarrow \mathscr{X}$ is called a generalized derivation if there exists a derivation (in the usual sense) $\delta: \mathscr{A} \rightarrow \mathscr{X}$ such that $\mu(a b)=a \mu(b)+\delta(a) b$ for all $a, b \in \mathscr{A}$. Familiar examples are the derivations from $\mathscr{A}$ to $\mathscr{X}$ and all so-called inner generalized derivations; those are defined by $\mu_{x, y}(a)=x a-a y$ for fixed arbitrary elements $x, y \in \mathscr{X}$. Moreover, every right multiplier (i.e., an additive map $h$ of $\mathscr{A}$ satisfying $h(a b)=a h(b)$ for all $a, b \in \mathscr{A})$ is a generalized derivation.

The stability of derivations was studied by Park in $[13,14]$. A discussion of the stability of the so-called $(\sigma-\tau)$-derivations and a study of the so-called generalized $(\theta, \phi)$ derivations are given in $[2,11]$, respectively. The present paper is devoted to the study of the stability of generalized derivations. The results of this paper are a generalization of those of Park's papers $[13,14]$.

Throughout the paper, $A$ denotes a unital normed algebra with unit 1 and $\mathscr{X}$ is a unitlinked Banach $\mathscr{A}$-bimodule in the sense that $1 x=x 1=x$ for all $x \in \mathscr{X}$.

\section{Main results}

Our aim is to establish the generalized Hyers-Ulam-Rassias stability of generalized derivations. We extend main results of Park [14] to generalized derivations from a unital 
normed algebra to a unit linked Banach $\mathscr{A}$-bimodule. We apply the direct method which was first devised by Hyers [7] to construct an additive function from an approximate one and use some ideas of $[11,13]$.

Theorem 2.1. Suppose $f: \mathscr{A} \rightarrow \mathscr{X}$ is a mapping with $f(0)=0$ for which there exist a map $g: \mathscr{A} \rightarrow \mathscr{X}$ and a function $\varphi: \mathscr{A} \times \mathscr{A} \times \mathscr{A} \times \mathscr{A} \rightarrow[0, \infty)$ such that

$$
\begin{gathered}
\tilde{\varphi}(a, b, c, d):=\frac{1}{2} \sum_{n=0}^{\infty} 2^{-n} \varphi\left(2^{n} a, 2^{n} b, 2^{n} c, 2^{n} d\right)<\infty \\
\|f(\lambda a+\lambda b+c d)-\lambda f(a)-\lambda f(b)-c f(d)-g(c) d\| \leq \varphi(a, b, c, d)
\end{gathered}
$$

for all $\lambda \in \mathbb{T}=\{\lambda \in \mathbb{C}:|\lambda|=1\}$ and all $a, b, c, d \in \mathscr{A}$. Then there exists a unique generalized derivation $\mu: \mathscr{A} \rightarrow \mathscr{X}$ such that

$$
\|f(a)-\mu(a)\| \leq \tilde{\varphi}(a, a, 0,0)
$$

for all $a \in \mathscr{A}$.

Proof. Setting $c=d=0$ and $\lambda=1$ in (2), we have

$$
\|f(a+b)-f(a)-f(b)\| \leq \varphi(a, b, 0,0)
$$

for all $a, b \in \mathscr{A}$. Now we use the Rassias method on inequality (2.4) (see $[5,10]$ ). One can use induction on $n$ to show that

$$
\left\|\frac{f\left(2^{n} a\right)}{2^{n}}-f(a)\right\| \leq \frac{1}{2} \sum_{k=0}^{n-1} 2^{-k} \varphi\left(2^{k} a, 2^{k} a, 0,0\right)
$$

for all $n \in \mathbb{N}$ and all $a \in \mathscr{A}$, and that

$$
\left\|\frac{f\left(2^{n} a\right)}{2^{n}}-\frac{f\left(2^{m} a\right)}{2^{m}}\right\| \leq \frac{1}{2} \sum_{k=m}^{n-1} 2^{-k} \varphi\left(2^{k} a, 2^{k} a, 0,0\right)
$$

for all $n>m$ and all $a \in \mathscr{A}$. It follows from the convergence (2.1) that the sequence $\left\{f\left(2^{n} a\right) / 2^{n}\right\}$ is Cauchy. Due to the completeness of $\mathscr{X}$, this sequence is convergent. Set

$$
\mu(a):=\lim _{n \rightarrow \infty} \frac{f\left(2^{n} a\right)}{2^{n}} .
$$

Putting $c=d=0$ and replacing $a, b$ by $2^{n} a, 2^{n} b$, respectively, in (2.2), we get

$$
\left\|2^{-n} f\left(2^{n}(\lambda a+\lambda b)\right)-2^{-n} \lambda f\left(2^{n} a\right)-2^{-n} \lambda f\left(2^{n} b\right)\right\| \leq 2^{-n} \varphi\left(2^{n} a, 2^{n} b, 0,0\right)
$$


4 Hyers-Ulam-Rassias stability of generalized derivations

Taking the limit as $n \rightarrow \infty$ we obtain

$$
\mu(\lambda a+\lambda b)=\lambda \mu(a)+\lambda \mu(b)
$$

for all $a, b \in \mathscr{A}$ and all $\lambda \in \mathbb{T}$.

Next, let $\gamma=\theta_{1}+\mathbf{i} \theta_{2} \in \mathbb{C}$ where $\theta_{1}, \theta_{2} \in \mathbb{R}$. Let $\gamma_{1}=\theta_{1}-\left[\theta_{1}\right]$, let $\gamma_{2}=\theta_{2}-\left[\theta_{2}\right]$. Then $0 \leq \gamma_{i}<1,(1 \leq i \leq 2)$ and by using [12, Remark 2.2.2] one can represent $\gamma_{i}$ as $\gamma_{i}=\left(\lambda_{i, 1}+\right.$ $\left.\lambda_{i, 2}\right) / 2$ in which $\lambda_{i, j} \in \mathbb{T}(1 \leq i, j \leq 2)$. Since $\mu$ satisfies (2.9) we infer that

$$
\begin{aligned}
\mu(\gamma x) & =\mu\left(\theta_{1} x\right)+\mathbf{i} \mu\left(\theta_{2} x\right) \\
& =\left[\theta_{1}\right] \mu(x)+\mu\left(\gamma_{1} x\right)+\mathbf{i}\left(\left[\theta_{2}\right] \mu(x)+\mu\left(\gamma_{2} x\right)\right) \\
& =\left(\left[\theta_{1}\right] \mu(x)+\frac{1}{2} \mu\left(\lambda_{1,1} x+\lambda_{1,2} x\right)\right)+\mathbf{i}\left(\left[\theta_{2}\right] \mu(x)+\frac{1}{2} \mu\left(\lambda_{2,1} x+\lambda_{2,2} x\right)\right) \\
& =\left(\left[\theta_{1}\right] \mu(x)+\frac{1}{2} \lambda_{1,1} \mu(x)+\frac{1}{2} \lambda_{1,2} \mu(x)\right)+\mathbf{i}\left(\left[\theta_{2}\right] \mu(x)+\frac{1}{2} \lambda_{2,1} \mu(x)+\frac{1}{2} \lambda_{2,2} \mu(x)\right) \\
& =\theta_{1} \mu(x)+\mathbf{i} \theta_{2} \mu(x)=\gamma \mu(x)
\end{aligned}
$$

for all $x \in \mathscr{A}$. So $\mu$ is $\mathbb{C}$-linear.

Moreover, it follows from (2.5) and (2.7) that $\|f(a)-\mu(a)\| \leq \tilde{\varphi}(a, a, 0,0)$ for all $a \in$ $\mathcal{A}$. It is known that additive mapping $\mu$ satisfying (2.3) is unique [1].

Putting $\lambda=1, a=b=0$, and replacing $c, d$ by $2^{n} c, 2^{n} d$, respectively, in (2.2) we obtain

$$
\left\|f\left(2^{2 n} c d\right)-2^{n} c f\left(2^{n} d\right)-2^{n} g\left(2^{n} c\right) d\right\| \leq \varphi\left(0,0,2^{n} c, 2^{n} d\right),
$$

whence

$$
\left\|2^{-2 n} f\left(2^{2 n} c d\right)-2^{-n} c f\left(2^{n} d\right)-2^{-n} g\left(2^{n} c\right) d\right\| \leq 2^{-2 n} \varphi\left(0,0,2^{n} c, 2^{n} d\right) .
$$

Put $d=1$ in (2.12). By (2.7), $\lim _{n \rightarrow \infty} 2^{-2 n} f\left(2^{2 n} a\right)=\mu(a)$ and by the convergence of series (2.1), $\lim _{n \rightarrow \infty} 2^{-2 n} \varphi\left(0,0,2^{n} c, 2^{n} d\right)=0$. Hence the sequence $\left\{2^{-n} g\left(2^{n} c\right)\right\}$ is convergent. Set $\delta(c):=\lim _{n \rightarrow \infty} 2^{-n} g\left(2^{n} c\right), c \in \mathscr{A}$. Let $n$ tend to $\infty$ in (2.12). Then

$$
\mu(c d)=c \mu(d)+\delta(c) d
$$

Next we claim that $\delta$ is a derivation. Put $d=1$ in (2.13). Then $\delta(c)=\mu(c)-c \mu(1)$. Hence $\delta$ is linear. Further,

$$
\begin{aligned}
\delta\left(c_{1} c_{2}\right) & =\mu\left(c_{1} c_{2}\right)-c_{1} c_{2} \mu(1) \\
& =\left(c_{1} \mu\left(c_{2}\right)+\delta\left(c_{1}\right) c_{2}\right)-c_{1} c_{2} \mu(1) \\
& =c_{1} \mu\left(c_{2}\right)+\left(\mu\left(c_{1}\right)-c_{1} \mu(1)\right) c_{2}-c_{1} c_{2} \mu(1) \\
& =c_{1}\left(\mu\left(c_{2}\right)-c_{2} \mu(1)\right)+\left(\mu\left(c_{1}\right)-c_{1} \mu(1)\right) c_{2} \\
& =c_{1} \delta\left(c_{2}\right)+\delta\left(c_{1}\right) c_{2} .
\end{aligned}
$$

Thus $\delta$ satisfies the Leibnitz rule. It then follows from (2.13) that $\mu$ is a generalized derivation. 
Remark 2.2. The significance of functional equation (2.2) is that the required derivation $\delta$ is naturally constructed. In other words, we do not need any additional functional inequality for existence of $\delta$.

Remark 2.3. As $\mathscr{A}$ is unital, the mapping $\delta$ that appeared in the definition of generalized derivation is unique. In fact, $\delta(a)=\mu(a)-a \mu(1)$.

Corollary 2.4. Suppose that $f: \mathscr{A} \rightarrow \mathscr{X}$ is a mapping with $f(0)=0$ for which there exist constants $\beta \geq 0$ and $0<p<1$ such that

$$
\|f(\lambda a+\lambda b+c d)-\lambda f(a)-\lambda f(b)-c f(d)-g(c) d\| \leq \beta\left(\|a\|^{p}+\|b\|^{p}+\|c\|^{p}+\|d\|^{p}\right)
$$

for all $\lambda \in \mathbb{T}$ and all $a, b, c, d \in \mathscr{A}$.

Then there is a unique generalized derivation $\mu: \mathscr{A} \rightarrow \mathscr{X}$ such that

$$
\|f(a)-\mu(a)\| \leq \frac{\beta\|a\|^{p}}{1-2^{p-1}}
$$

for all $a \in \mathscr{A}$.

Proof. Put $\varphi(a, b, c, d)=\beta\left(\|a\|^{p}+\|b\|^{p}+\|c\|^{p}+\|d\|^{p}\right)$ in Theorem 2.1.

Proposition 2.5. Suppose that $f: \mathscr{A} \rightarrow \mathscr{X}$ is a mapping with $f(0)=0$ for which there exists a function $\varphi: \mathscr{A} \times \mathscr{A} \times \mathscr{A} \times \mathscr{A} \rightarrow[0, \infty)$ such that

$$
\begin{gathered}
\tilde{\varphi}(a, b, c, d):=\frac{1}{2} \sum_{n=0}^{\infty} 2^{-n} \varphi\left(2^{n} a, 2^{n} b, 2^{n} c, 2^{n} d\right)<\infty, \\
\|f(\lambda a+\lambda b+c d)-\lambda f(a)-\lambda f(b)-c f(d)-g(c) d\| \leq \varphi(a, b, c, d)
\end{gathered}
$$

for $\lambda=1, \mathbf{i}$ and for all $a, b, c, d \in \mathscr{A}$. If for each fixed $a \in \mathscr{A}$ the function $t \mapsto f(t a)$ is continuous on $\mathbb{R}$, then there exists a unique generalized derivation $\mu: \mathscr{A} \rightarrow \mathscr{X}$ such that $\| f(a)-$ $\mu(a) \| \leq \tilde{\varphi}(a, a, 0,0)$ for all $a \in \mathscr{A}$.

Proof. Put $c=d=0$ and $\lambda=1$ in (2.2). It follows from the proof of Theorem 2.1 that there exists a unique additive mapping $\mu: \mathscr{A} \rightarrow \mathscr{X}$ given by $\mu(a)=\lim _{n \rightarrow \infty}\left(f\left(2^{n} a\right) / 2^{n}\right)$, $a \in \mathcal{A}$. By the same reasoning as in the proof of the theorem of [15], the mapping $\mu$ is $\mathbb{R}$-linear.

Assuming $b=c=d=0$ and $\lambda=\mathbf{i}$, it follows from (2.2) that $\|f(\mathbf{i} a)-\mathbf{i} f(a)\| \leq$ $\varphi(a, 0,0,0), a \in \mathcal{A}$. Hence $\left(1 / 2^{n}\right)\left\|f\left(2^{n} \mathbf{i} a\right)-\mathbf{i} f\left(2^{n} a\right)\right\| \leq \varphi\left(2^{n} a, 0,0,0\right)$ for all $n \in N$ and $a \in \mathscr{A}$. The right-hand side tends to zero as $n \rightarrow \infty$ so that

$$
\mu(\mathbf{i} a)=\lim _{n \rightarrow \infty} \frac{f\left(2^{n} \mathbf{i} a\right)}{2^{n}}=\lim _{n \rightarrow \infty} \frac{i f\left(2^{n} a\right)}{2^{n}}=\mathbf{i} \mu(a)
$$

for all $a \in \mathscr{A}$. For each $\lambda \in \mathbb{C}, \lambda=r_{1}+\mathbf{i} r_{2}\left(r_{1}, r_{2} \in \mathbb{R}\right)$. Hence

$$
\begin{aligned}
\mu(\lambda a) & =\mu\left(r_{1} a+\mathbf{i} r_{2} a\right)=r_{1} \mu(a)+r_{2} \mu(\mathbf{i} a) \\
& =r_{1} \mu(a)+\mathbf{i} r_{2} \mu(a)=\left(r_{1}+\mathbf{i} r_{2}\right) \mu(a)=\lambda \mu(a) .
\end{aligned}
$$


Thus $\mu$ is $\mathbb{C}$-linear. The fact that $\mu$ is a generalized derivation can be deduced in the same fashion as in the proof of Theorem 2.1.

Proposition 2.6. Let $\mathscr{A}$ be a unital $C^{*}$-algebra. Suppose that $f: \mathscr{A} \rightarrow \mathscr{X}$ is a mapping with $f(0)=0$ for which there exists a function $\varphi: \mathscr{A} \times \mathscr{A} \times \mathscr{A} \times \mathscr{A} \rightarrow[0, \infty)$ such that

$$
\begin{gathered}
\tilde{\varphi}(a, b, c, d):=\frac{1}{2} \sum_{n=0}^{\infty} 2^{-n} \varphi\left(2^{n} a, 2^{n} b, 2^{n} c, 2^{n} d\right)<\infty \\
\|f(\lambda a+\lambda b+c d)-\lambda f(a)-\lambda f(b)-c f(d)-g(c) d\| \leq \varphi(a, b, c, d), \\
\left\|f\left(2^{n} u^{*}\right)-f\left(2^{n} u\right)^{*}\right\| \leq \varphi\left(2^{n} u, 2^{n} u, 0,0\right)
\end{gathered}
$$

for all $\lambda \in \mathbb{T}$, all $a, b, c, d \in \mathscr{A}$, all nonnegative integers $n$, and all unitaries $u$ in $\mathcal{A}$. Then there exists a unique generalized derivation $\mu: \mathscr{A} \rightarrow \mathscr{X}$ such that $\|f(a)-\mu(a)\| \leq \tilde{\varphi}(a, a, 0,0)$ for all $a \in \mathscr{A}$.

Proof. It follows from the proof of Theorem 2.1 that there exists a unique generalized derivation $\mu: \mathscr{A} \rightarrow \mathscr{X}$ given by $\mu(a)=\lim _{n \rightarrow \infty}\left(f\left(2^{n} a\right) / 2^{n}\right), a \in \mathscr{A}$ satisfying (2.3).

Using (2.20), we have

$$
\left\|2^{-n} f\left(2^{n} u^{*}\right)-2^{-n} f\left(2^{n} u\right)^{*}\right\| \leq 2^{-n} \varphi\left(2^{n} u, 2^{n} u, 0,0\right) .
$$

Letting $n \rightarrow \infty$ we conclude that $\mu\left(u^{*}\right)=\mu(u)^{*}$. Since $\mu$ is linear and every element of a $C^{*}$-algebra can be represented as a linear combination of unitaries [12], we deduce that $\mu\left(a^{*}\right)=\mu(a)^{*}$.

Now let $\mathscr{A}$ be a unital Banach algebra. The mapping $f: \mathscr{A} \rightarrow \mathscr{A}$ is called an approximately generalized derivation if $f(0)=0$ and there exist a positive number $\varepsilon$ and a mapping $g: \mathscr{A} \rightarrow \mathscr{A}$ such that

$$
\|f(\lambda a+\lambda b+c d)-\lambda f(a)-\lambda f(b)-c f(d)-g(c) d\| \leq \varepsilon
$$

for all $\lambda \in \mathbb{T}$ and all $a, b, c, d \in \mathscr{A}$.

Theorem 2.7. Let $\mathscr{A}$ be a unital Banach algebra and let $f: \mathscr{A} \rightarrow \mathscr{A}$ be an approximately generalized derivation with the corresponding mapping $g$. Then $f$ is a generalized derivation and $g$ is a derivation.

Proof. Put $\varphi(a, b)=\varepsilon$ in Theorem 2.1. Then we get a generalized derivation $\mu$ defined by $\mu(a):=\lim _{n \rightarrow \infty}\left(f\left(2^{n} a\right) / 2^{n}\right)$ such that

$$
\|\mu(a)-f(a)\| \leq \varepsilon
$$


for all $a \in \mathscr{A}$. We have

$$
\begin{aligned}
\left\|2^{n}\left(f\left(2^{m} a\right)-2^{m} f(a)\right)\right\| \leq & \left\|2^{n} 1 f\left(2^{m} a\right)-g\left(2^{n} 1\right) 2^{m} a-f\left(\left(2^{n} 1\right)\left(2^{m} a\right)\right)\right\| \\
& +\left\|f\left(\left(2^{n} 1\right)\left(2^{m} a\right)\right)-g\left(2^{n} 1\right) 2^{m} a-2^{n+m} 1 f(a)\right\| \\
\leq & \varepsilon+\left\|f\left(\left(2^{n} 1\right)\left(2^{m} a\right)\right)-g\left(2^{n} 1\right) 2^{m} a-2^{n+m} 1 f(a)\right\| \\
\leq & \varepsilon+\left\|f\left(\left(2^{n} 1\right)\left(2^{m} a\right)\right)-\mu\left(\left(2^{n} 1\right)\left(2^{m} a\right)\right)\right\| \\
& +\left\|\mu\left(\left(2^{n} 1\right)\left(2^{m} a\right)\right)-2^{n+m} 1 f(a)-g\left(2^{n} 1\right) 2^{m} a\right\| \\
\leq & 2 \varepsilon+\left\|\mu\left(\left(2^{n} 1\right)\left(2^{m} a\right)\right)-2^{n+m} 1 f(a)-g\left(2^{n} 1\right) 2^{m} a\right\| \\
\leq & 2 \varepsilon+2^{m}\left\|\mu\left(2^{n} 1 a\right)-f\left(2^{n} 1 a\right)\right\| \\
& +2^{m}\left\|f\left(2^{n} 1 a\right)-2^{n} 1 f(a)-g\left(2^{n} 1\right) a\right\| \\
\leq & \left(2+2^{m+1}\right) \varepsilon
\end{aligned}
$$

for all nonnegative integers $m, n$ and all $a \in \mathscr{A}$. Fix $m$ and let $n$ tend to $\infty$ in the following inequality:

$$
\left\|f\left(2^{m} a\right)-2^{m} f(a)\right\| \leq \frac{2+2^{m+1}}{2^{n}} \varepsilon .
$$

Then $f\left(2^{m} a\right)=2^{m} f(a)$ for all $m$ and all $a \in \mathscr{A}$. Therefore $\mu(a)=\lim _{m \rightarrow \infty}\left(f\left(2^{m} a\right) / 2^{m}\right)=$ $f(a)$ for all $a \in \mathscr{A}$.

\section{Acknowledgment}

The author would like to thank the referees for their useful comments.

\section{References}

[1] C. Baak and M. S. Moslehian, On the stability of J*-homomorphisms, Nonlinear Analysis. Theory, Methods \& Applications 63 (2005), no. 1, 42-48.

$[2] \_$, Generalized $(\theta, \phi)$-derivations on Banach algebras, preprint.

[3] S. Czerwik (ed.), Stability of Functional Equations of Ulam-Hyers-Rassias Type, Hadronic Press, Florida, 2003.

[4] Z. Gajda, On stability of additive mappings, International Journal of Mathematics and Mathematical Sciences 14 (1991), no. 3, 431-434.

[5] P. Găvruța, A generalization of the Hyers-Ulam-Rassias stability of approximately additive mappings, Journal of Mathematical Analysis and Applications 184 (1994), no. 3, 431-436.

[6] B. Hvala, Generalized derivations in rings, Communications in Algebra 26 (1998), no. 4, 11471166.

[7] D. H. Hyers, On the stability of the linear functional equation, Proceedings of the National Academy of Sciences of the United States of America 27 (1941), 222-224.

[8] M. Mathieu (ed.), Elementary Operators \& Applications, World Scientific, New Jersey, 1992, Proceedings of the International Workshop.

[9] M. Mirzavaziri and M. S. Moslehian, Automatic continuity of $\sigma$-derivations in $C^{*}$-algebras, to appear in Proceedings of the American Mathematical Society. 
[10] M. S. Moslehian, Approximately vanishing of topological cohomology groups, Journal of Mathematical Analysis and Applications 318 (2006), no. 2, 758-771.

[11] Approximate $(\sigma-\tau)$-contractibility, to appear in Nonlinear Functional Analysis and Applications.

[12] G. J. Murphy, $C^{*}$-Algebras and Operator Theory, Academic Press, Massachusetts, 1990.

[13] C.-G. Park, Lie *-homomorphisms between Lie $C^{*}$-algebras and Lie *-derivations on Lie $C^{*}$ algebras, Journal of Mathematical Analysis and Applications 293 (2004), no. 2, 419-434.

[14] Linear derivations on Banach algebras, Nonlinear Functional Analysis and Applications 9 (2004), no. 3, 359-368.

[15] Th. M. Rassias, On the stability of the linear mapping in Banach spaces, Proceedings of the American Mathematical Society 72 (1978), no. 2, 297-300.

[16] Th. M. Rassias (ed.), Functional Equations, Inequalities and Applications, Kluwer Academic, Dordrecht, 2003.

[17] Th. M. Rassias and P. Šemrl, On the behavior of mappings which do not satisfy Hyers-Ulam stability, Proceedings of the American Mathematical Society 114 (1992), no. 4, 989-993.

[18] S. M. Ulam, Problems in Modern Mathematics, chapter VI, Science Editions, John Wiley \& Sons, New York, 1964.

Mohammad Sal Moslehian: Department of Mathematics, Ferdowsi University, P.O. Box 1159, Mashhad 91775, Iran; Banach Mathematical Research Group (BMRG), Mashhad, Iran E-mail address: moslehian@ferdowsi.um.ac.ir 


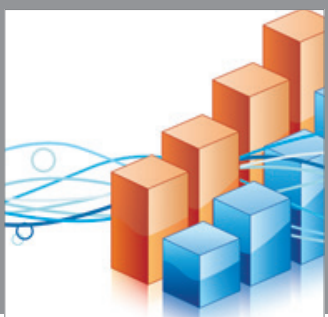

Advances in

Operations Research

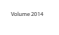

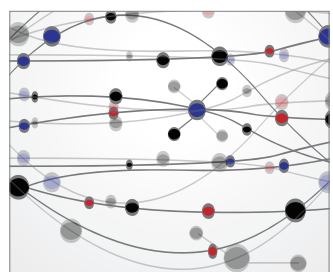

\section{The Scientific} World Journal
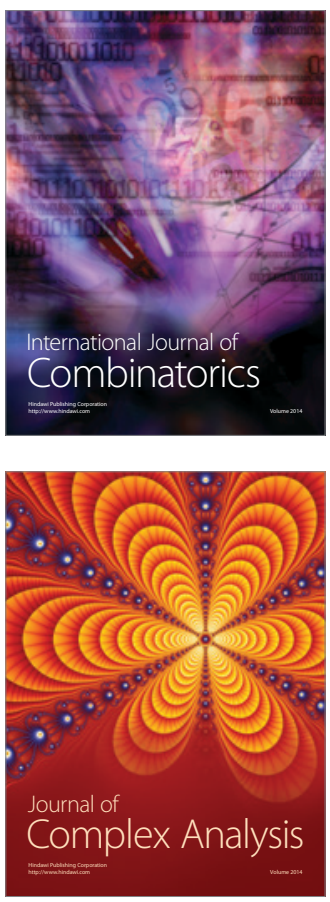

International Journal of

Mathematics and

Mathematical

Sciences
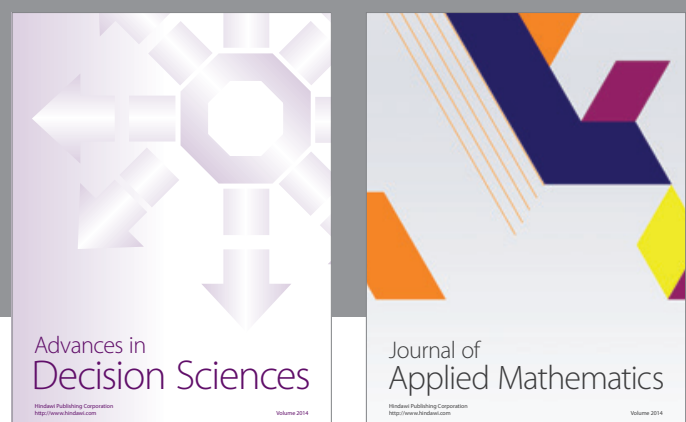

Journal of

Applied Mathematics
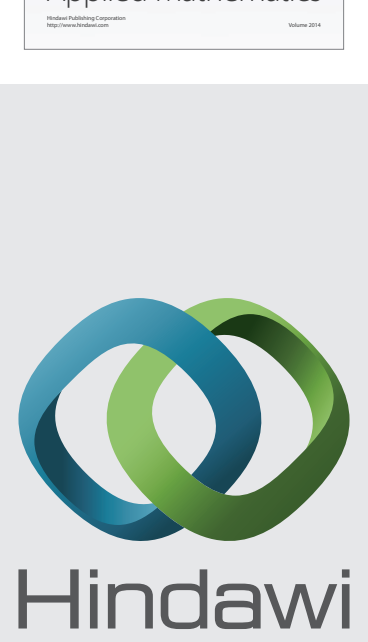

Submit your manuscripts at http://www.hindawi.com
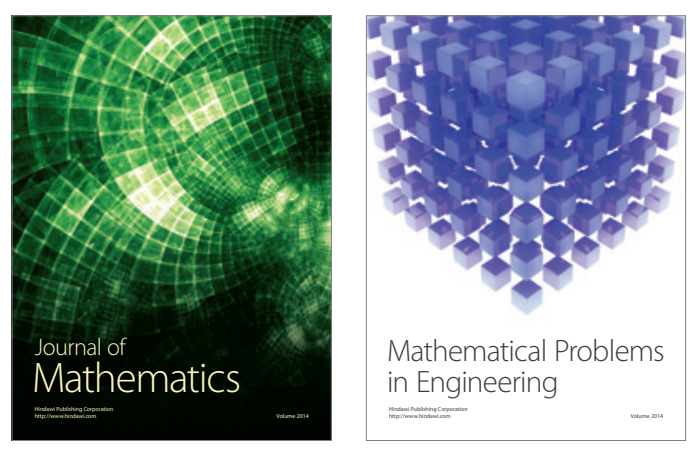

Mathematical Problems in Engineering
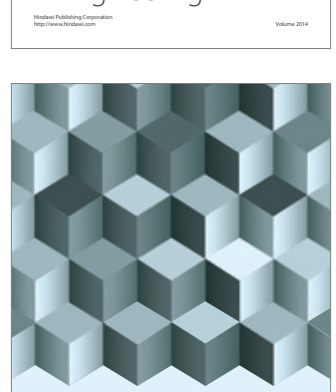

Journal of

Function Spaces
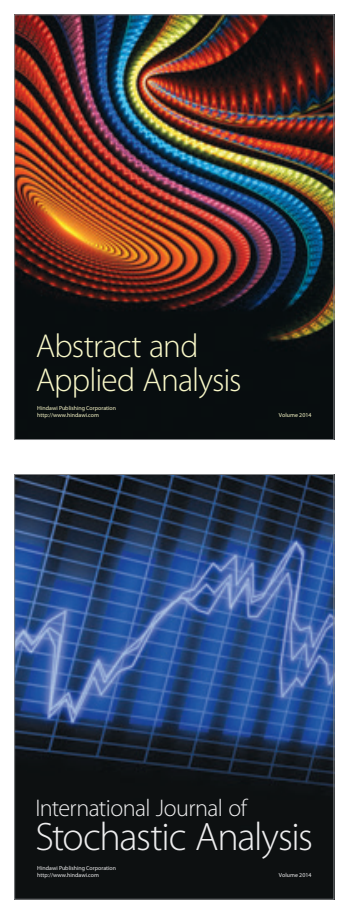

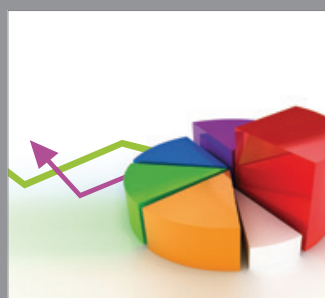

ournal of

Probability and Statistics

Promensencen
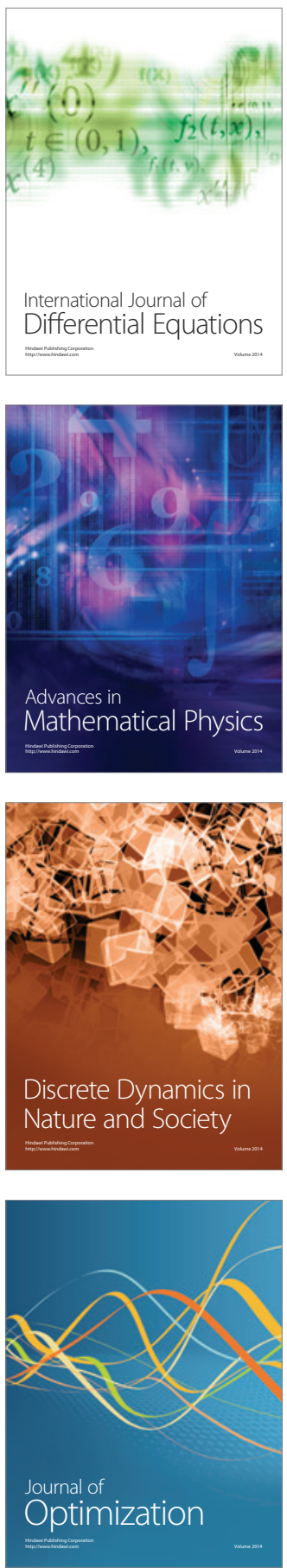